\title{
Absence probable de la translocation robertsonienne $1 / 29$ en race bovine Blanc Bleu Belge
}

\author{
N Nicolas ${ }^{1}$, A Hidas ${ }^{2}$, R Renaville ${ }^{1}$, R Kettmann ${ }^{1}, \mathrm{~N} \mathrm{Gengler}^{3}$, \\ A Burny ${ }^{1}, \mathrm{~F}$ Boonen ${ }^{4}$, J Toszer ${ }^{2}$, A Goffinet ${ }^{1}$ \\ ${ }^{1}$ Faculté des sciences agronomiques, UER biologie moléculaire et physiologie animale, \\ avenue Maréchal-Juin, 13, 5030 Gembloux, Belgique; \\ ${ }^{2}$ Université des sciences agricoles, Institut de recherche pour les petits animaux, \\ laboratoire de cytogénétique, 2105 Gödölló, Hongrie; \\ ${ }^{3}$ Faculté des sciences agronomiques, UER zootechnie, passage des Déportés, 2, \\ 5030 Gembloux; \\ ${ }^{4}$ Centre de sélection bovine, rue des Champs-Élysées, 5590 Ciney, Belgique
}

(Reçu le 28 Avril 1994; accepté le 6 Avril 1995)

\begin{abstract}
Résumé - La translocation robertsonienne 1/29, anomalie chromosomique de structure, intervient fréquemment comme cause d'infertilité. Nous avons donc voulu connaître sa fréquence en race bovine Blanc Bleu Belge, jusqu'alors non étudiée pour ce facteur. Au sein d'un échantillon de 138 taureaux de race Blanc Bleu Belge, la translocation 1/29 n'a pas pu être identifiée. Nous pouvons estimer avec $5 \%$ de risque d'erreur que la fréquence de cette translocation au sein de la population Blanc Bleu Belge doit être inférieure à $0,69 \%$. Ce résultat trouve une explication dans l'origine de cette race. En effet, le Blanc Bleu Belge est issu d'un mélange de races laitières locales et de la race Shorthorn, lesquelles peuvent être a priori considérées comme indemnes de la translocation 1/29.
\end{abstract}

translocation robertsonienne 1/29 / race bovine Blanc Bleu Belge / infertilité

Summary - Probable absence of the 1/29 Robertsonian translocation in the Belgian Blue breed of cattle. The 1/29 translocation, a structural chromosomal abnormality, is often associated with infertility. For this reason, we wished to know its frequency in the Belgian Blue breed. In this study, none of the 138 Belgian Blue bulls karyotyped carried the 1/29 translocation. We calculated that the 1/29 translocation frequency in the Belgian Blue breed must be lower than $0.69 \%$ at the $5 \%$ level. This result can be explained by the origins of the breed. The Belgian Blue breed is descended from local dairy breeds and from the Shorthorn breed, which can all be considered to be free of the 1/29 translocation.

1/29 Robertsonian translocation / Belgian Blue cattle / infertility 


\section{INTRODUCTION}

Parmi les différentes causes d'infertilité, les anomalies chromosomiques sont responsables d'un nombre considérable de mortalités embryonnaires (Popescu, 1990). Chez le bovin, la translocation robertsonienne 1/29 (t 1/29), anomalie de structure, est l'une des plus largement répandues (Gustavsson et Rockborn, 1964).

La t $1 / 29$ résulte de la jonction centromérique de 2 chromosomes acrocentriques non homologues appartenant aux paires 1 et 29 , à la suite de 2 cassures en-deçà et au-delà du centromère et formant un seul chromosome submétacentrique. Ceci aboutit à la réduction du nombre total de chromosomes à 59 chez les porteurs hétérozygotes et à 58 chez les porteurs homozygotes au lieu de $60 \mathrm{chez}$ les bovins normaux (Gary et al, 1991). Cependant, les gamètes à caryotype déséquilibré gardent leur capacité de fécondation normale et donnent des embryons non viables, monosomiques ou trisomiques, pour l'un ou l'autre des 2 chromosomes impliqués dans l'anomalie.

Bien qu'identifiée dans une cinquantaine de races bovines avec une fréquence variant entre 0 et $70 \%$ en fonction du nombre d'animaux examinés (Foulley et Frebling, 1985; Long, 1985; Popescu et Pech, 1991), seules 2 races, la Pie-rouge Suédoise et la Blonde d'Aquitaine ont fait l'objet d'une étude systématique sur un échantillon représentatif de plus de 2000 animaux. Les observations réalisées ont montré que plus de $14 \%$ des femelles sont hétérozygotes porteuses de la translocation (Gustavsson, 1969; Frebling et al, 1987).

Compte tenu des informations bibliographiques relatées ci-avant, nous avons entrepris l'étude de la t 1/29 en race Blanc Bleu Belge (BBB) pour laquelle aucune donnée n'existe actuellement.

\section{MATÉRIEL ET MÉTHODES}

\section{Animaux}

Un échantillon sanguin $(10 \mathrm{ml})$ a été prélevé sur 138 taureaux culards de race BBB provenant du Centre de sélection bovine de Ciney (Belgique) $(n=113)$, du Centre d'insémination artificielle Haliba (Mons, Belgique) $(n=23)$ et de 2 exploitations agricoles situées en province de Namur (Belgique) $(n=2)$. Le sang a été recueilli sur héparine-sodium et conservé à $4{ }^{\circ} \mathrm{C}$ jusqu'aux analyses.

\section{Méthode analytique}

L'analyse cytogénétique des échantillons a été réalisée par microculture. Après homogénéisation, $1 \mathrm{ml}$ de sang est additionné de $2,5 \mathrm{ml}$ de milieu de culture Parker TC-199 ou RPMI 1640 (Gibco) supplémenté de 20\% de sérum foetal de veau, $1 \%$ de L-Glutamine, $2 \%$ de Pénicilline-Streptomycine (Gibco), de l'héparine-sodium $(5 \mathrm{UI} / \mathrm{ml})$ et $1 \%$ de Pokeweed mitogen (Gibco). Les cultures sont incubées à $38^{\circ} \mathrm{C}$ pendant $72 \mathrm{~h}$.

Les divisions cellulaires sont arrêtées au stade métaphase par ajout d'une goutte de vinblastine $(0,5-1 \mathrm{mg}$ par $\mathrm{ml}$ total de culture). Après $1 \mathrm{~h}$ d'incubation et un 
choc hypotonique dans du chlorure de potassium $(0,56 \%)$, les chromosomes sont fixés dans un mélange éthanol absolu-acide acétique glacial (3:1) pendant une nuit.

Après étalement sur lame, les suspensions cellulaires sont colorées avec du Giemsa à $4 \%$ et un minimum de 5 métaphases sont examinées au microscope (Leitz Diaplan) en contraste de phase.

\section{RÉSULTATS}

Aucun des 138 taureaux BBB étudiés n'est porteur de la translocation robertsonienne $1 / 29$. L'absence de la translocation $1 / 29$ dans cette étude ne permet toutefois pas de conclure définitivement quant à la fréquence de la t 1/29 dans la population totale $\mathrm{BBB}$. Les méthodes relatives aux proportions, en particulier la transformation angulaire (Dagnelie, 1986), permettent d'estimer pour un niveau de signification donné, la fréquence maximale attendue. Au niveau de signification 0,05 , la fréquence de la $\mathrm{t} 1 / 29$ au sein de la population $\mathrm{BBB}$ est estimée inférieure ou égale à $0,69 \%$.

\section{DISCUSSION}

La technique cytogénétique utilisée (coloration au Giemsa) est limitée à l'étude d'un cas particulier de translocation réciproque, la fusion de type robertsonienne entre 2 chromosomes acrocentriques. D'autres anomalies de structure telles que l'inversion ou la translocation réciproque s'opposent également au déroulement normal de la méiose et ne peuvent pas être observées par cette technique.

À ce jour, une cinquantaine de races bovines, essentiellement européennes (Gustavsson, 1979), ont été identifiées comme porteuses de la t 1/29 (Popescu et Pech, 1991). Il n'est toutefois pas exclu que d'autres races soient porteuses de cette anomalie.

En races laitières, la t 1/29 n'a pas été observée chez la Holstein-Frisonne, la Jersey, la Normande et l'Ayrshire (Eldridge, 1985; Foulley et Frebling, 1985; Long, 1985).

Parmi les races à viande, la translocation robertsonienne, objet de cette étude, s'est révélée être absente dans plusieurs races largement échantillonnées comme la Maine-Anjou, l'Angus, l'Hereford et la Shorthorn (Eldridge, 1985; Long, 1985 ; Schmutz et al, 1990; Nel et al, 1991). Toutefois, Gustavsson (1979), Foulley et Frebling (1985), Nel et al (1991) et Vainas et al (1992) ont rapporté que la fréquence de la t 1/29 était plus élevée dans les races à viande que dans les races laitières. Nel et al (1991) estiment que la faible représentation ou l'absence de la t 1/29 en races laitières peut être expliquée par les pratiques d'élevage utilisées en race pure et selon lui, par la sélection constante pour la fertilité pratiquée dans de telles races.

La translocation 1/29 est absente de notre échantillon de 138 taureaux de race Blanc Bleu Belge. Ce résultat peut trouver une explication dans les origines de la race $B B B$, à savoir un mélange de gènes de races laitières locales auxquelles a été infusé du sang Shorthorn. En effet, les études relatées ci-avant montrent l'absence de la t 1/29 en race Shorthorn et, selon les auteurs cités, la t 1/29 est, par comparaison aux races à viande, moins présente dans les races laitières. 
Enfin, compte tenu qu'un faible lien de parenté entre les animaux étudiés (maximum $9 \%$ ) établi sur 3 générations a été mesuré et compte tenu du fait que les animaux analysés représentent $31 \%$ de la population des centres de sélection et d'insémination, nous pouvons conclure au terme de notre étude que la probabilité d'observer la t 1/29 en race Blanc Bleu Belge est très faible et en tout cas vraisemblablement inférieure à $0,69 \%$.

\section{REMERCIEMENTS}

Nous remercions vivement MM F Boonen, C Boccart, M Dive, E Marchand et A Toussaint pour leur participation à la collecte des échantillons sanguins. Nous remercions également M M Lardau pour son aide lors de l'obtention du fichier pedigree.

Nous tenons également à remercier M I Szalay, directeur de l'Institut de recherche pour les petits animaux de Gödölló (Hongrie) pour son accueil ainsi que tout le personnel du laboratoire. R Kettmann est directeur de recherches du FNRS et N Gengler est aspirant du FNRS.

\section{RÉFÉRENCES}

Dagnelie P (1986) Théorie et méthodes statistiques. Applications agronomiques (tome II). Presses agron, Gembloux, Belgique

Eldridge FE (1985) Cytogenetics of Livestock. AVI Publishing Company, Inc, Westport, CT.

Foulley JL, Frebling J (1985) La translocation 1-29 chez les bovins : distribution, effets, procédure d'éradication. Bull Tech CRZV Theix, INRA 62, 93-102

Frebling J, Foulley JL, Berland HM, Popescu CP, Cribiu EP, Darré R (1987) Résultats de l'enquête sur la fréquence de la translocation 1/29 en race bovine Blonde d'Aquitaine. Bull Tech CRZV Theix, INRA 67, 49-58

Gary F, Berland HM, Berthelot X, Darré R (1991) La translocation robertsonienne 1/29 chez les bovins : intérêt du dépistage et des mesures d'éradication. Le point vétérinaire $22,851-856$

Gustavsson I (1969) Cytogenetics distribution and phenotypic effects of a translocation in Swedish cattle. Hereditas 63, 68-169

Gustavsson I (1979) Distribution and effects of the 1/29 Robertsonian translocation in cattle. J Dairy Sci 62, 825-835

Gustavsson I, Rockborn G (1964) Chromosome abnormality in 3 cases of lymphatic leukemia in cattle. Nature 203, 990

Long SE (1985) Centric fusion translocations in cattle: a review. Vet Rec 116, 516-518

Nel ND, Harris EJ, Weiermans JE, Meyer EHH (1991) The cytogenetic screening of South African artificial insemination bulls. Genet Sel Evol 23, 109-117

Popescu CP (1990) Conséquences des anomalies de la structure chromosomique chez les animaux domestiques. Reprod Nutr Dev suppl 1, 105s-116s

Popescu CP, Pech A (1991) Une bibliographie sur la translocation 1/29 de bovins dans le monde (1964-1990). Ann Zootech 40, 271-305

Schmutz SM, Moker JS, Barth AD, Mapletoft RJ (1990) Embryonic loss in superovulated cattle caused by the 1/29 Robertsonian translocation. Theriogenology 35, 705-714

Vainas E, Belibasakis N, Boscos C (1992) Robertsonian translocation in Sykia Challkidiki cattle. Theriogenology 37, 1085-1089 